\title{
Prenatal Cocaine Exposure Affects Social Behavior in Sprague- Dawley Rats
}

\author{
Josephine M. Johns ${ }^{1}$ and Linda R. Noonan \\ Brain and Development Research and Department of Psychiatry, The University of North \\ Carolina at Chapel Hill, Chapel Hill, NC 27599
}

\begin{abstract}
Children prenatally exposed to cocaine are reported to exhibit inappropriate social behavior, including aggression. We have recently observed a similar phenomenon in rats prenatally exposed to cocaine. Pregnant females were injected twice daily with $15 \mathrm{mg} / \mathrm{kg}$ cocaine hydrochloride or saline on gestation days 1-20. Offspring were tested for social behavior towards two unfamiliar, untreated rats of the same age and sex. Cocaine-treated males (90 PND) took longer to reciprocate contact and cocaine-treated females (60 PND) spent more time rough grooming unfamiliar females. Male cocaine offspring (180 PND) tested for aggression exhibited an increased frequency and duration and decreased latency to chase an intruder. ACTH was lower in cocaine-treated males (150-180 PND) following plus-maze exposure or exposure to an unfamiliar male. Our data indicate that prenatal cocaine treatment in rats increases fear or aggression responses, dependent on sex and stimulus situation.
\end{abstract}

\section{Keywords}

Cocaine; Prenatal; Development; Social behavior; Aggression; ACTH; HPA axis

The Alarming rise of cocaine abuse among pregnant women has promoted much concern over the potential behavioral effects on prenatally exposed neonates and infants $(4,20)$. Offspring of mothers that have abused cocaine during pregnancy have exhibited signs of increased irritability and altered state lability as newborns $(2,3)$. Research has also shown that young children prenatally exposed to cocaine are somewhat aggressive, show poor social attachment, and display abnormal play behavior in unstructured environments $(7,18)$. These data are suggestive of a cocaine-induced, abnormal development of socioemotional behavior. However, mothers who have abused cocaine during pregnancy have been shown to bond poorly to their infants (1), making it difficult to determine if the origin of these deficits occur as a direct result of cocaine acting on the developing fetus, being raised in an unstable or abusive environment in which parental drug abuse may be prevalent, or a combination thereof.

Recent animal research on the effects of prenatal cocaine exposure has begun to focus on behaviors that appear to be similar to the behaviors exhibited by human offspring prenatally exposed to cocaine. Rodent studies suggest that offspring exposed prenatally to cocaine exhibit signs of behavioral abnormalities including increased "emotionality" and neophobia $(5,8)$, and aggression towards an intruder (9). Though these studies employed cross-fostered

Copyright (C) 1995 Elsevier Science Inc. Printed in the USA. All rights reserved

${ }^{1}$ Requests for reprints should be addressed to Josephine M. Johns, Ph.D., BDRC CB\#7250, UNC School of Medicine, Chapel Hill, NC 27599. 
offspring, another recent study found that untreated offspring fostered to cocaine-treated mothers also displayed higher levels of shock-elicited aggression (6), suggesting a possible postnatal environmental influence.

Based on the previous findings of abnormal behavioral responses of fear and aggression in male rats upon reaching puberty or adulthood, respectively $(8,9)$, the present study was undertaken to determine if these kinds of behaviors changes were task or sex related. We hypothesized that the appearance of the behaviors would be age related and that females as well as males would show abnormal behavioral development following exposure to prenatal cocaine. To test these hypotheses, cross-fostered male and female offspring of mothers treated with cocaine during pregnancy were examined for abnormal responses of fear and aggression across several developmental ages.

\section{METHOD}

\section{General Methodology}

Nulliparous Sprague-Dawley female rats (Charles River, Raleigh, NC) were randomly assigned to a cocaine, saline, or surrogate group on the morning a sperm plug was found (designated as gestational day 0). Saline and cocaine dams received SC injections twice daily throughout gestation (CD 1-20), at approximately 0900 and $1630 \mathrm{~h}$, of normal saline or $15 \mathrm{mg} / \mathrm{kg}$ of cocaine hydrogen chloride (Sigma Chemical Co., St. Louis, MO) dissolved in distilled water. Surrogates received no treatment during gestation. They were housed singly on a $12 \mathrm{~L}: 12 \mathrm{D}$ cycle with the light period beginning at $0800 \mathrm{~h}$. Surrogate and cocaine treatment dams had free access to food (rat chow) and all subjects had free access to water. Saline-treated females were matched for date of pregnancy, age, and weight with a chronic cocaine treatment dam and received the same amount of rat chow on each day of gestation as had been consumed by its matched cocaine dam on the same day of gestation. Mean weight gain during pregnancy, gestational length, number of live pups delivered, and litter weight are reported in Table 1. A portion of the offspring born to surrogate mothers was designated as the untreated group for social behavioral testing. Saline, cocaine, and untreated offspring were cross-fostered, in litters of 8-10 pups, to untreated, surrogate mothers within $24 \mathrm{~h}$ of birth. Pups were weaned on postnatal day 21 (birth = PND 0). After weaning, pups from each litter were randomly selected for behavioral testing at 60,90 , or 180 days of age and housed in same-sex groups of three. Each animal was tested at only one age and on one behavioral test.

\section{Open Field}

One male and female rat from each saline and cocaine litter were randomly selected for open field testing at 90 days postnatal. Figure 1 represents the open field apparatus. The procedure used for this study has been previously described (8). Briefly, the start box was identical to the home cage except that it contained no bedding. The start box and open field were made of translucent plastic and joined by a $10-\mathrm{cm}$ internal diameter, $12-\mathrm{cm}$ long PVC pipe tunnel. As shown in Fig. 1, the floor of the apparatus was marked off into nine rectangles of equal area. Subjects were removed from their home cages and placed in the start box. Subjects were given $5 \mathrm{~min}$ to leave the start box and traverse the tunnel into the open field. Two front paws across a grid line signified entry into a new grid. Frequency, latency to enter, and duration in each grid section and reentries to the start box were recorded for a 10-min period on a laptop computer. If an animal failed to enter the open field apparatus within $5 \mathrm{~min}$, it was removed from the start box and placed into grid section 1 facing the center of the open field and the observation begun. Data were collected between 0900 and $1630 \mathrm{~h}$, with all groups represented during morning and afternoon hours. 


\section{Social Behavior}

One male and one female from each of the cocaine, saline, and untreated litters were randomly selected for observation of social behavior. Males were tested at 90 and females tested at 60 days of age. Subjects were marked for identification and placed in a large open field apparatus $(69.5 \times 67 \mathrm{~cm})$ with two untreated animals of the same age and sex. Untreated animals were used in only one session. The 10-min test session was videotaped and subsequently scored for frequency, duration, and latency of nine interactive social behaviors exhibited by the experimental animal [see Table 2; behaviors were taken in part from $(12,13,17)]$. Data were collected between 0900 and $1630 \mathrm{~h}$, with all groups represented during morning and afternoon hours.

\section{Aggressive Behavior}

Two males from each of the prenatal treatment group litters were randomly selected for aggression testing, one at 90 and the other at 180 days of age. A resident intruder model similar to that used previously by Miczek (14) was employed. Each subject was housed separately with an ovariectomized female in a large polypropylene cage $(59 \times 39 \times 20 \mathrm{~cm}$.) for weeks. Females were removed $1 \mathrm{~h}$ before testing and males were injected subcutaneously with $0.9 \%$ normal saline 30 min prior to testing. Food and water were removed from the cage and the cage was placed in a small observation room. A smaller intruder male rat (250-275 g) was placed into the cage with the subject. Frequency, duration, and latency for each of the behaviors described in Table 3 were recorded over a 15 -min period [behaviors were taken in part from $(12,13,17)$ ]. Data were recorded between 0900 and $1630 \mathrm{~h}$ with all groups represented during morning and afternoon hours. Intruder males were used in only one session.

\section{Plus-Maze}

Eleven males from cocaine and 12 males from saline treatment litters were tested in the elevated plus-maze between the ages of 150-180 days postnatal. Approximately half of the males from each treatment group were naive (no previous testing) and the other half had previously served as the control group for a drug experiment not included in the present study. These animals were observed in a home cage for behavior 1 month prior to the plusmaze testing and had no observable effects on the plus-maze data. The elevated plus-maze consisted of four $8-\mathrm{cm}$ wide by $50-\mathrm{cm}$ long arms intersecting at right angles. Two of the arms were enclosed by 40-cm high walls and two were open. The maze was elevated $1 \mathrm{~m}$ above the floor in a room with standard fluorescent lighting. Subjects were started at the center of the maze. Time spent in the open arms, the closed arms, and number of crosses from any arm into any other arm were measured during a 5-min test period. Entry into an arm was defined as the head and both forepaws inside the arm. Plus-maze testing occurred between 1200 and $1630 \mathrm{~h}$.

\section{ACTH and Corticosterone}

To assess the activity of the hypothalamo-pituitary-adrenal (HPA) axis, we measured ACTH and corticosterone in serum from 150-180-day-old males. Serum was obtained by tail vein puncture at 0 and 90 min following the mild novelty stress of the plus-maze testing previously described. ACTH was also measured in treatment males 90 min following placement for $15 \mathrm{~min}$ in the home cage of an unfamiliar, equal size male. All blood samples were collected between 1200 and $1630 \mathrm{~h}$. Unfamiliar males were used in only one session. For 2 days prior to testing, the animals were habituated to all aspects of the blood collection procedure (transport, general restraint, tail immersion in warm water to produce vasodilation) except venous puncture. ACTH and corticosterone were assayed using commercial ${ }^{125}$ I radioimmunoassay kits from Incstar and ICN Biomedical, respectively. The 
ACTH antibody sensitivity was $15 \mathrm{pg} / \mathrm{ml}$ and it was $100 \%$ cross-reactive with peptide sequences 1-24 and 1-39; cross-reactivity with $\alpha-\mathrm{MSH}$ and $\beta$-endorphin was less than $0.01 \%$. The corticosterone antibody sensitivity was $3 \mathrm{pg} / \mathrm{ml}$ and cross-reacted less than $0.50 \%$ with desoxycorticosterone and less than $0.10 \%$ with all other steroids.

\section{Statistical Analysis}

The Fisher's exact probability test was used to assess differences in the number of animals per group that displayed a particular behavior (24). Frequency, duration, and latency data as well as hormonal measures were analyzed with analyses of variance (ANOVAs). Statistical significance was assumed at values of $p \leq 0.05$.

\section{RESULTS \\ Gestational Data}

There were no significant differences between untreated, saline-, and cocaine-treated dams on measures of gestational weight gain, gestational length, number of live pups, or litter weight (Table 1).

\section{Open Field}

There were no significant differences with respect to sex within groups or treatment between groups on any open field measure at 90 days of age. One of 10 cocaine-treated offspring (a male) and 2 of 10 saline offspring (one male, one female) failed to enter the start box within the first $5 \mathrm{~min}$ of testing. The mean latency to enter the apparatus from the start box was slightly longer (nonsignificant) for cocaine-treated offspring (58.22 \pm 19.9$)$ than for salinetreated offspring $(45.43 \pm 9.4)$. Cocaine-treated offspring also had a slightly longer duration $(246.58 \pm 29.1)$ in the start box than did saline-treated offspring $(182.6 \pm 29.1)$ throughout the test period (nonsignificant).

\section{Social Behavior}

Saline and untreated males were combined into a single control group for statistical comparison with cocaine-treated males as their behavioral measures were not significantly different statistically from one another. The combined saline and untreated group is referred to as "control" in Tables 4-6. Cocaine males tested at 90 days of age took significantly longer to reciprocate contact (Table 6) with the unfamiliar males than did control males, $F(1,15)=7.09, p<0.02$.

Saline and untreated females were combined into a single control group for statistical comparison with cocaine-treated females as their results were not significantly different from one another. Cocaine-exposed females tested at 60 days of age rough groomed unfamiliar conspecifics more than did control females, $F(1,15)=4.70, p<0.05$ (Table 4). Cocaine-treated females exhibited a somewhat longer duration of rough grooming and a longer latency before allowing contact, though data were nonsignificant (both $p<0.08$ ). Tables 4, 5, and 6 present data for males and females on all measures of social behavior.

\section{Aggression}

There were no significant treatment effects at 90 days of age, although all of the cocainetreated males (six of six) as opposed to two of five of the saline-treated males rough groomed the intruder male for $150 \mathrm{~s}$ or more of the 15 -min test period (nonsignificant). Mean frequency, duration, and latency of aggressive behaviors in 90-day males are presented in Tables 7, 8, and 9. 
At 180 days of age, only 3 of 10 saline treated males compared to 10 of 11 cocaine-treated males chased the intruder male (Fisher's exact, $p<0.01$ ). Cocaine-treated males chased the intruder male more often, $F(1,19)=11.04$, p < 0.01, for a longer duration, $F(1,19)=12.36$, $p<0.01$, and with a shorter latency, $F(1,19)=10.55, p<0.01$, than did saline-treated males (Tables 7-9).

\section{Plus-Maze}

There were no significant differences between saline and cocaine males on time spent in the open or closed arms of the maze (anxiety measure) nor total crosses (locomotor measure). The saline and cocaine groups, respectively, spent (mean \pm SEM) $45.3 \pm 10.0$ and $41.2 \pm 7.3$ $\mathrm{s}$ in the open arms and $188.6 \pm 12.5$ vs. $186 \pm 16.6 \mathrm{~s}$ in the closed arms. The number of crosses were $10.2 \pm 1.4$ and $8.0 \pm 1.1$.

For females it was observed that cocaine animals spent slightly more time in the open arms of the plus maze when compared with saline animals $F(1,28)=4.78, p<.05$. The number of total crosses made and time spent in the closed arms of the plus maze did not significantly differ between these groups. The saline and cocaine females respectively spent $30.8 \pm 6.4$ versus $50.8 \pm 6.1 \mathrm{~s}$ in the open arms, and $202.8 \pm 11.8 \mathrm{vs} .177 .9 \pm 9.1 \mathrm{~s}$ in the closed arms of the plus maze. The number of crosses were $10.6 \pm 1.3$ and $12.6 \pm 0.8$.

\section{Hormonal Measures}

Both ACTH, $F(1,44)=25.87, p<0.01$, and corticosterone, $F(1,44)=8.01, p<0.01$, were lower at $90 \mathrm{~min}$ than at $0 \mathrm{~min}$ following exposure of males to the plus-maze. ACTH levels were significantly decreased at both 0 - and 90-min test points in cocaine-treated males compared to saline-treated males following plus-maze exposure, $F(1,44)=4.84, p<0.05$ (Fig. 2). Corticosterone levels did not differ significantly between saline- and cocainetreated males. Mean corticosterone values for saline and cocaine males, respectively, were $142.8 \pm 11.8$ and $163.8 \pm 8.6$ at $0 \mathrm{~min}$ and $106.0 \pm 15.2$ vs. $120.7 \pm 18.7$ at $90 \mathrm{~min}$. Ninety minutes following exposure to an unfamiliar male, cocaine-treated males exhibited a significant decrease in ACTH levels when compared to saline-treated males, $F(1,21)=5.40$, $p<0.05$.

There were no significant effects of prenatal cocaine treatment on female ACTH or corticosterone, although there was a trend for ACTH to be lower in cocaine females at 90 min following plus maze exposure. The ACTH levels $(\mathrm{pg} / \mathrm{ml})$ for female saline and cocaine animals are, repsectively, $121.0 \pm 12.2$ vs. $134.5 \pm 17.3$ at $0 \mathrm{~min}$ and $105.2 \pm 13.8$ versus $84.4 \pm 6.4$ at $90 \mathrm{~min}$ following the plus maze. Corticosterone levels $(\mathrm{ng} / \mathrm{ml})$ for female saline and cocaine animals are, respectively, $472.0 \pm 71.3$ versus $502.0 \pm 24.0$ at $0 \mathrm{~min}$, and 376.0 \pm 23.8 vs. $419.0 \pm 29.7$ at $90 \mathrm{~min}$ following the plus maze.

\section{DISCUSSION}

Our data, taken in conjunction with previous data on responses to novel environments, in particular the open field situation (8), suggest that in male rats prenatally exposed to cocaine there is a pattern of abnormal responses to novel situations both with and without other animals present. This study did not find increased latency to enter a novel open field at PND 90, though this has been shown to occur in male offspring at PND 60 (8). Cocaine-induced fear responses in novel nonsocial situations appear to be age related as significant differences between saline- and cocaine-exposed male offspring disappear sometime between 60 and 90 days of age, slightly earlier than what we observed in social testing situation. 
We did not observe any significant effects of prenatal cocaine in the plus-maze. It was hypothesized that 150-180-day-old males would exhibit more anxiety or fear on this test, consistent with the data from younger males on open field (8) and social behavior tests. Whereas it is possible that the cocaine-induced increase in fear disappears somewhere between 90 and 150 days, it appears more likely that the plus-maze task is relatively insensitive to the subtle behavioral differences that occur as a result of prenatal cocaine treatment. The plus-maze was originally designed to test anxiolytic drugs that produce large increases in open arm visitation. Our saline animals decidedly preferred the closed arms of the maze and chose to spend only $14 \%$ of their time in the open arms, making a further decrease in open arm time in cocaine males more difficult to detect. Because we did not conduct plus-maze testing during early adolescence, no conclusions can be drawn concerning if there might be deficits in this behavior at an early age.

The pattern of inappropriate cocaine-induced responses that appears around adolescence as increased fear and avoidance (8) later takes the form of increased aggression (9). There were no differences between groups in females on novelty responding at 90 days of age, but at 60 days of age the cocaine-exposed females respond to a social situation with a slight increase in rough grooming (mildly aggressive). It may be that prenatal cocaine exposure somehow alters the offspring's sensory, perceptual, or arousal capabilities such that a stimulus appears more threatening to the cocaine offspring and they respond with fear or aggression, depending on their age, sex, and testing environment.

This study both replicates and extends the results of previous research that reported a cocaine-induced increase in male aggression at 180 PND (9). In agreement with previous research (6), we did not observe significant increases in aggression at $90 \mathrm{PND}$, although there was a trend toward more rough grooming of the intruder at this age. Rough grooming is a moderately aggressive behavior that we have observed in pilot studies that frequently precedes fighting in older males tested in this paradigm. This may indicate an emerging behavioral pattern that becomes more robust at 180 PND, when the resident intruder model of aggression is used. In contrast, when tested in the social situation that was not specifically designed to elicit aggression, fear or avoidance appears to be the more prevalent response in males at PND 90. PND 90 may be the transitional age at which cocaine-exposed male offspring exhibit both fear and aggression in a social situation, dependent on the context. More data using a larger sample from PND 90 are needed to state with more certainty that cocaine-induced aggression is not present and only beginning to appear at this age.

Male and female cocaine-treated animals had a somewhat different behavioral profile. Cocaine-treated females displayed more mildly aggressive behavior towards other females in the social situation at 60 PND whereas the males were less likely to be aggressive in the intruder male model until some-time after 90 PND. Unfortunately, we did not have a task specifically designed to elicit aggression in females as we did for the males. Recently, we discovered that cocaine treatment during pregnancy results in significantly higher rates of aggression by a dam towards an intruder male when tested with pups present (10). Future studies will determine the usefulness of this maternal aggression model for assessing female aggression following prenatal cocaine exposure.

Differences between cocaine- and saline-treated offspring do not appear to be the result of maternal gestational abnormalities. There were no significant treatment differences on the indices of normal gestation measured in this study. There have been concerns about whether skin lesions caused by daily cocaine injections given chronically could cause some of the behavioral differences seen in offspring. We have used this particular paradigm on a number of occasions and have found that dividing the treatment into two cocaine injections in twice the volume of distilled water $(2 \mathrm{ml} / \mathrm{kg}$ ) significantly reduces both the severity and frequency 
of lesions. Only a few dams developed lesions at all, most of which occurred during the final week of gestation and were easily treated with a topical antibiotic (polymycin, bacitracin, neomycin, Burroughs Wellcome Co.). A study conducted on this issue specifically for the National Institutes of Drug Abuse indicated that there were are no apparent behavioral or biochemical indications of significant stress to the dam due directly to the lesions (16). One could also argue that the frequency of injections was stressful, but because saline controls received the same number of injections the effects should have been similarly distributed across groups.

Because cocaine-treated males appear to overreact behaviorally to novel testing situations, we hypothesized that the activity of the HPA axis would also be increased in response to mild stress. However, the observed decrease in ACTH levels in both a social and nonsocial novel situation suggests that the HPA axis may have been permanently altered by the prenatal stress of cocaine administration to the mother. Acute cocaine administration to adult rats is probably stressful and has been shown to elevate ACTH and corticosterone levels (15), which in the pregnant rat may affect the developing fetus. Repeated corticosterone administration or repeated stress to the pregnant mother has been shown to reduce HPA axis activity in the offspring (19,21). Decreased HPA axis (cortisol) activity has also been reported in human infants prenatally exposed to cocaine (11). Alternatively, it is possible that decreased ACTH levels in cocaine-exposed rats may represent the effect of increased negative feedback and the HPA's attempt to achieve homeostasis in the face of a chronic elevation in corticosterone. We did not observe significant hypercorticosteronemia in the cocaine animals, though an episodic hypersecretion may have been missed by the sampling times chosen. Another possibility is that the presumed compensatory decrease in ACTH was sufficient and effective to return corticosterone levels to within their normal range. A naturalistic approach to this latter hypothesis would be to measure basal and stress-induced levels of corticosterone in infant or aged animal, both of which have less efficient or diminished negative feedback function.

We have observed some subtle albeit replicable increases in fear and aggression in offspring of mothers treated with cocaine during pregnancy. These behavioral changes may be a result of cocaine acting directly on the fetal CNS, or cocaine-induced vasoconstriction during pregnancy and resultant hypoxic conditions for the fetus or even abnormal infant/mother or littermate interactions. Behavioral changes in cocaine-treated offspring may begin early in life, while the pup is still in the nest, and could change the caretaking behavior of the mothers. There is also evidence for prenatal cocaine-induced suppression of weanling play behavior (23), which has been shown to influence subsequent adult social interactions (22). We suggest that prenatal cocaine exposure results in an overall enhanced reactivity to environmental stimuli that can be observed as alterations in behavior throughout development by tests sensitive to these particular alterations. Observation of prenatal cocaine-induced changes may depend on designing tests that are age appropriate.

\section{Acknowledgments}

We would like to thank Dr. Cort Pedersen for providing the breeding animals necessary for this project. We would also like to thank Ms. Li Li and Ms. Betsy Shambley for technical assistance and help in manuscript preparation. This research was supported by a NICHD Mental Retardation Interdisciplinary Training Program Fellowship T32HG07201 (J.M.J.), Foundation of Hope for Treatment and Research in Mental Illness, National Alliance for Research in Schizophrenia and Depression, and NIMH R29-MH46442 (L.R.N.).

\section{REFERENCES}

1. Burns K, Chethik L, Burns WJ, Clark R. Dyadic disturbances in cocaine-abusing mothers and their infants. J. Clin. Psychol. 1991; 313:666-669. 
2. Chasnoff IJ, Burns KA, Burns WJ. Cocaine use in pregnancy: Perinatal morbidity and mortality. Neurotoxicol. Teratol. 1987; 9:291-293. [PubMed: 3683346]

3. Chasnoff IJ, Burns WJ, Schnoll SH, Burns KA. Cocaine use in pregnancy. N. Engl. J. Med. 1985; 313:666-669. [PubMed: 4022059]

4. Chasnoff IJ, Landress HJ, Barrett ME. The prevalence of illicit-drug or alcohol use during pregnancy and discrepancies in mandatory reporting in Pinellas County, Florida. N. Engl. J. Med. 1990; 322:1202-1206. [PubMed: 2325711]

5. Church MW, Overbeck GW. Prenatal cocaine exposure in the Long-Evans rat: II. Dose-dependent effects on offspring behavior. Neurotoxicol. Teratol. 1990; 12:327-334. [PubMed: 2392091]

6. Goodwin GA, Heyser CJ, Moody CA, Rajachandran L, Molina VA, Arnold HM, McKinzie DL, Spear NE, Spear LP. A fostering study of the effects of prenatal cocaine exposure: II. Offspring behavioral measures. Neurotoxicol. Teratol. 1992; 14:423-432. [PubMed: 1488037]

7. Howard J, Beckwith L, Rodnig C, Kropenske V. The development of young children of substanceabusing parents: Insights from seven years of intervention and research. Zero to Three. 1989; 9:812.

8. Johns JM, Means MJ, Anderson DR, Bass EW, Means LW, McMillen BA. Prenatal exposure to cocaine II: Effects on open field activity and cognitive behavior in Sprague-Dawley rats. Neurotoxicol. Teratol. 1992; 14:343-349. [PubMed: 1454043]

9. Johns JM, Means LW, Bass EW, Means MJ, Zimmerman LI, McMillen BA. Prenatal exposure to cocaine: Effects on aggression in Sprague-Dawley rats. Dev. Psychobiol. 1994; 27:227-239. [PubMed: 7913451]

10. Johns JM, Noonan LR, Zimmerman LI, Li L, Pedersen CA. Effects of chronic and acute cocaine treatment on the onset of maternal behavior and aggression in Sprague-Dawley rats. Behav. Neurosci. 1994; 108:1-7.

11. Magnano CL, Gardner JM, Karmel BZ. Differences is salivary cortisol levels in cocaine-exposed and noncocaine exposed NICU infants. Dev. Psychobiol. 1992; 25:93-103. [PubMed: 1577206]

12. Means LW, Sydow M, DaVanzo JP. The effect olfactory bulb lesions on agonistic, locomotor and social behavior in mice housed in aggregate. Physiol. Behav. 1984; 32:657-661. [PubMed: 6541352]

13. Miczek KA. Intraspecies aggression in rats: Effects of d-amphetamine and chlordiazepoxide. Psychopharmacologia. 1974; 39:275-301. [PubMed: 4476082]

14. Miczek KA. Effects of 1-dopa, d-amphetamine and cocaine on intruder evoked aggression in rats and mice. Neuropsychopharmacology. 1977; 1:271-277.

15. Moldow RL, Fischman AJ. Cocaine-induced secretion of ACTH, beta-endorphin, and corticosterone. Peptides. 1987; 8:819-822. [PubMed: 2829143]

16. National Institute on Drug Abuse. Bowman Gray study on the effects of subutaneous injections or oral administration of cocaine HCL or cocaine Mel on pregnancy in Sprague-Dawley rats. Rockville, MD: National Institute on Drug Abuse; 1993.

17. Olivier B. Selective anti-aggressive properties of DU 27725: Ethological analyses of intermale and territorial aggression in the male rat. Pharmacol. Biochem. Behav. 1981; 14:61-77. [PubMed: 6894643]

18. Oro AS, Dixon SD. Perinatal cocaine and methamphetamine exposure: Maternal and neonatal correlates. J. Pediatr. 1987; 111:571-578. [PubMed: 3655989]

19. Pollard I. Effects of stress administered during pregnancy on reproductive capacity and subsequent development of the offspring in rats: Prolonged effects on the litter of a second pregnancy. J. Endocrinol. 1984; 100:301-306. [PubMed: 6699535]

20. Schutzman DL, Frankenfield-Chernicoff M, Clatterbaugh HE, Singer J. Incidence of intrauterine cocaine exposure in a suburban setting. Pediatrics. 1991; 88:825-827. [PubMed: 1896293]

21. Skebelskaya YB. The effect of corticosteroid concentrations in the blood of gravid rats on the adrenocorticotropic function of hypophysis of the fetus. Gen. Comp. Endocrinol. 1968; 10:263268. [PubMed: 4297499]

22. Taylor GT. Fighting in juvenile rats and the ontogeny of agonistic behavior. J. Comp. Physiol. Psychol. 1980; 94:953-961. 
23. Wood RD, Bannoura MD, Johanson IB. Prenatal cocaine exposure: Effects on play behavior in the juvenile rat. Neurotoxicol. Teratol. 1994; 16:139-144. [PubMed: 8052187]

24. Zar, JH. Biostatistical analysis. Prentice Hall, Inc.: Englewood Cliffs, NJ; 1984. 


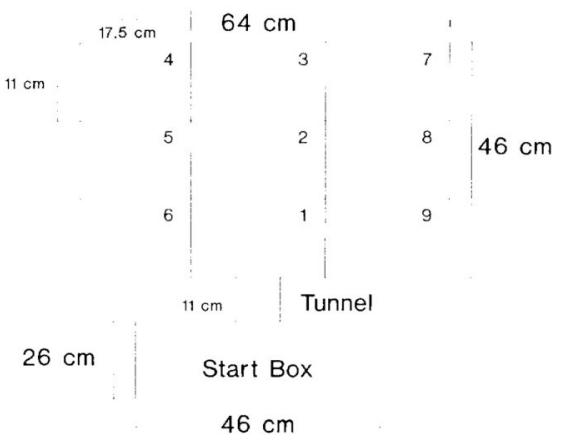

FIG. 1.

Open field apparatus. 




FIG. 2.

Serum ACTH levels were significantly lower in males prenatally exposed to cocaine compared to saline-exposed males, at 0 and 90 min following the mild stress of plus-maze exposure, and 90 min following exposure to an unfamiliar male. 




Neurotoxicol Teratol. Author manuscript; available in PMC 2011 June 7. 
TABLE 2

Behavioral categories and descriptions (Social Behavior)

\begin{tabular}{ll}
\hline Behavior & Description \\
\hline Contact other & The focal animal touches another rat with his nose. \\
Reciprocal contact & Focal rat touches another rat which has contacted his body. \\
Rough groom & Focal rat roughly grooms other rat around neck, back, or genital region. \\
Chase & Pursuit of other rat around the arena. \\
Bite & Focal rat bites one or both of the other rats. \\
Escape & Focal rat is moving away from one or both of the other rats that are in pursuit. \\
Avoid contact & Focal rat repulses contact from other rats by pushing away, turning, or moving a short distance. \\
Allow contact & Focal rat is passive to contact by other rats. \\
Other & Focal rat displays any behavior other than those listed. \\
\hline
\end{tabular}


TABLE 3

Behavioral categories and descriptions (Aggression)

\begin{tabular}{|c|c|}
\hline Behavior & Description \\
\hline Alert position & Focal rat exhibits a sudden interruption of all movement with head directed towards stimulus animal. \\
\hline Piloerection & Focal rat exhibits hair fluffing/piloerection. \\
\hline Aggressive posture & Intruder is in supine position. Focal rat restrains intruder with front paws. \\
\hline Fight attack & $\begin{array}{l}\text { A quick lunge by the focal rat usually followed by rolling, biting, and fur pulling directed towards the neck and back } \\
\text { regions. }\end{array}$ \\
\hline Chase & Pursuit of the intruder rat around the cage. \\
\hline Self-groom & Resident grooms itself by licking body fur and face washing, most often after a fight attack. \\
\hline Circle threat & Focal rat turns it's flank toward intruder with his back arched and legs extended, moving laterally against intruder. \\
\hline Defensive/submissive & Focal rat lies supine. Intruder posture restrains focal rat with front paws. \\
\hline Escape & Focal rat is moving away from intruder that is in pursuit. \\
\hline Rough groom & Focal rat roughly grooms the intruder around neck, back, and genital region. \\
\hline
\end{tabular}


TABLE 4

Frequency of social behaviors in male and female cocaine-treated and control offspring

\begin{tabular}{lccccr}
\hline & \multicolumn{2}{c}{ Females } & & \multicolumn{2}{c}{ Males } \\
\cline { 2 - 3 } \cline { 6 - 6 } Behavior Frequency & Cocaine (6) & Control (11) & & Cocaine (6) & Control (11) \\
\hline Reciprocate contact & $12.50 \pm 2.66$ & $11.00 \pm 2.42$ & & $7.50 \pm 2.60$ & $12.64 \pm 1.65$ \\
Avoid contact & $3.66 \pm 1.28$ & $4.63 \pm 1.28$ & & $5.17 \pm 1.35$ & $9.09 \pm 1.71$ \\
Allow contact & $5.00 \pm 1.24$ & $7.09 \pm 1.03$ & & $4.33 \pm 1.74$ & $6.00 \pm 0.59$ \\
Chase & $2.83 \pm 1.19$ & $3.63 \pm 1.34$ & & $5.33 \pm 2.35$ & $5.91 \pm 1.17$ \\
Rough groom & $5.83 \pm 2.12^{*}$ & $1.72 \pm 0.82$ & $6.50 \pm 2.19$ & $4.91 \pm 1.36$ \\
Move away & $11.00 \pm 2.76$ & $13.36 \pm 2.80$ & $4.67 \pm 0.49$ & $5.00 \pm 0.76$ \\
Contact other & $27.83 \pm 2.62$ & $20.72 \pm 2.53$ & & $22.17 \pm 3.36$ & $27.18 \pm 2.46$ \\
Punch bite & $0.00 \pm 0.00$ & $0.00 \pm 0.00$ & & $0.33 \pm 0.21$ & $0.36 \pm 0.20$ \\
\hline
\end{tabular}

Values are means \pm SEM and the number of subjects per group is shown in parentheses. * $p<0.05$. 
TABLE 5

Duration of social behaviors in male and female cocaine-treated and control offspring

\begin{tabular}{lrrrrr}
\hline & \multicolumn{2}{c}{ Females } & & \multicolumn{2}{c}{ Males } \\
\cline { 2 - 3 } Behavior Duration & Cocaine (6) & Control (11) & & Cocaine (6) & Control (11) \\
\hline Reciprocate contact & $49.00 \pm 11.02$ & $39.09 \pm 10.43$ & & $21.90 \pm 7.17$ & $32.96 \pm 8.33$ \\
Avoid contact & $11.00 \pm 5.40$ & $12.45 \pm 4.25$ & & $9.23 \pm 2.76$ & $20.85 \pm 5.79$ \\
Allow contact & $17.17 \pm 5.26$ & $28.09 \pm 5.49$ & & $15.15 \pm 8.91$ & $21.40 \pm 3.21$ \\
Chase & $7.00 \pm 3.24$ & $11.60 \pm 4.44$ & $21.07 \pm 14.14$ & $16.05 \pm 3.71$ \\
Rough groom & $21.66 \pm 10.71$ & $5.36 \pm 3.19$ & $28.22 \pm 11.10$ & $15.25 \pm 7.85$ \\
Move away & $46.17 \pm 16.57$ & $41.63 \pm 9.68$ & & $13.97 \pm 3.21$ & $17.54 \pm 4.01$ \\
Contact other & $116.67 \pm 18.06$ & $98.72 \pm 9.33$ & $87.82 \pm 14.81$ & $93.93 \pm 10.12$ \\
Punch bite & $0.00 \pm 0.00$ & $0.00 \pm 0.00$ & & $1.73 \pm 1.23$ & $0.92 \pm 0.60$ \\
\hline
\end{tabular}

Values are means \pm SEM and the number of subjects per group is shown in parentheses. 
TABLE 6

Latency of social behaviors in male and female cocaine-treated and control offspring

\begin{tabular}{lrrrrr}
\hline & \multicolumn{2}{c}{ Females } & & \multicolumn{2}{c}{ Males } \\
\cline { 2 - 3 } \cline { 6 - 6 } Behavior Latency & \multicolumn{1}{c}{ Cocaine (6) } & Control (11) & & Cocaine (6) & Control (11) \\
\hline Reciprocate contact & $66.17 \pm 25.84$ & $101.00 \pm 47.12$ & & $133.62 \pm 44.36^{*}$ & $34.90 \pm 13.86$ \\
Avoid contact & $267.83 \pm 75.41$ & $199.45 \pm 44.83$ & & $77.93 \pm 16.28$ & $93.60 \pm 24.45$ \\
Allow contact & $232.00 \pm 61.34$ & $122.09 \pm 27.07$ & & $93.52 \pm 31.80$ & $142.00 \pm 35.33$ \\
Chase & $262.00 \pm 103.79$ & $284.60 \pm 80.47$ & & $182.62 \pm 93.80$ & $112.69 \pm 50.54$ \\
Rough groom & $210.17 \pm 96.70$ & $399.91 \pm 66.84$ & & $188.58 \pm 85.03$ & $152.09 \pm 52.36$ \\
Move away & $125.33 \pm 59.92$ & $67.18 \pm 16.96$ & & $63.00 \pm 12.27$ & $194.49 \pm 48.72$ \\
Contact other & $13.00 \pm 2.80$ & $40.36 \pm 19.51$ & & $10.88 \pm 3.22$ & $28.95 \pm 16.27$ \\
Punch bite & $0.00 \pm 0.00$ & $0.00 \pm 0.00$ & & $511.58 \pm 86.39$ & $455.09 \pm 64.71$ \\
\hline
\end{tabular}

Values are means \pm SEM and the number of subjects per group is shown in parentheses.

* $p<0.02$ 
TABLE 7

Frequency of aggressive behaviors in 90- and 180-day-old male cocaine-treated and control offspring

\begin{tabular}{lrrrrr}
\hline & \multicolumn{2}{c}{ 90 PND } & & \multicolumn{2}{c}{ 180 PND } \\
\cline { 2 - 3 } \cline { 5 - 6 } Behavior Frequency & Cocaine (6) & Control (5) & & Cocaine (11) & Control (10) \\
\hline Other & $23.67 \pm 0.99$ & $18.20 \pm 1.93$ & & $20.82 \pm 2.23$ & $17.30 \pm 2.18$ \\
Alert posture & $0.83 \pm 0.31$ & $0.20 \pm 0.20$ & & $1.27 \pm 0.24$ & $0.90 \pm 0.18$ \\
Piloerection & $0.00 \pm 0.00$ & $0.00 \pm 0.00$ & $0.82 \pm 0.38$ & $1.10 \pm 0.38$ \\
Aggressive posture & $2.17 \pm 1.97$ & $0.00 \pm 0.00$ & & $1.09 \pm 0.58$ & $0.60 \pm 0.40$ \\
Fight attack & $1.83 \pm 1.64$ & $0.00 \pm 0.00$ & $1.36 \pm 0.89$ & $0.20 \pm 0.20$ \\
Chase & $0.67 \pm 0.67$ & $1.00 \pm 1.00$ & & $3.36 \pm 0.87^{*}$ & $0.30 \pm 0.15$ \\
Self-groom & $2.67 \pm 0.71$ & $2.00 \pm 0.84$ & $3.45 \pm 0.69$ & $3.80 \pm 0.76$ \\
Circle threat & $0.50 \pm 0.34$ & $0.00 \pm 0.00$ & & $0.55 \pm 0.37$ & $0.20 \pm 0.20$ \\
Defensive posture & $0.00 \pm 0.00$ & $0.00 \pm 0.00$ & & $0.09 \pm 0.09$ & $0.10 \pm 0.10$ \\
Escape & $0.17 \pm 0.17$ & $0.20 \pm 0.20$ & & $0.18 \pm 0.12$ & $0.80 \pm 0.36$ \\
Rough groom & $21.67 \pm 0.88$ & $16.20 \pm 2.84$ & $18.40 \pm 2.07$ & $14.20 \pm 2.25$ \\
\hline
\end{tabular}

Values are means \pm SEM and the number of subjects per group is shown in parentheses.

* $p<0.01$. 
TABLE 8

Duration of aggressive behaviors in 90- and 180-day-old male cocaine-treated and control offspring

\begin{tabular}{lrrrrr}
\hline & \multicolumn{2}{c}{ 90 PND } & & \multicolumn{2}{c}{ 180 PND } \\
\cline { 2 - 3 } Behavior Duration & Cocaine (6) & Control (5) & & Cocaine (11) & Control (10) \\
\hline Other & $594.03 \pm 35.65$ & $661.38 \pm 15.63$ & & $674.93 \pm 20.06$ & $659.77 \pm 32.02$ \\
Alert posture & $11.98 \pm 7.42$ & $0.76 \pm 0.76$ & & $7.73 \pm 1.61$ & $4.81 \pm 1.19$ \\
Piloerection & $0.00 \pm 0.00$ & $0.00 \pm 0.00$ & & $4.35 \pm 2.76$ & $13.97 \pm 11.24$ \\
Aggressive posture & $17.02 \pm 11.55$ & $0.00 \pm 0.00$ & & $6.51 \pm 4.09$ & $3.77 \pm 2.76$ \\
Fight attack & $4.43 \pm 3.42$ & $0.00 \pm 0.00$ & & $2.75 \pm 1.93$ & $0.21 \pm 0.21$ \\
Chase & $0.83 \pm 0.83$ & $2.00 \pm 2.00$ & & $10.62 \pm 2.29 *$ & $1.62 \pm 0.90$ \\
Self-groom & $29.13 \pm 10.48$ & $27.00 \pm 9.89$ & & $49.04 \pm 10.45$ & $51.52 \pm 13.60$ \\
Circle threat & $2.43 \pm 1.62$ & $0.00 \pm 0.00$ & & $2.33 \pm 1.47$ & $1.79 \pm 1.79$ \\
Defensive posture & $0.00 \pm 0.00$ & $0.00 \pm 0.00$ & & $0.15 \pm 0.15$ & $0.22 \pm 0.22$ \\
Escape & $0.28 \pm 0.28$ & $1.24 \pm 1.24$ & & $0.68 \pm 0.53$ & $3.77 \pm 1.84$ \\
Rough groom & $197.38 \pm 15.34$ & $143.40 \pm 30.99$ & $142.65 \pm 22.80$ & $156.39 \pm 25.75$ \\
\hline
\end{tabular}

Values are means \pm SEM and the number of subjects per group is shown in parentheses.

* $p<0.01$ 
TABLE 9

Latency of aggressive behaviors in 90- and 80-day-old male cocaine-treated and control offspring

\begin{tabular}{lrrrrr}
\hline & \multicolumn{2}{c}{ 90 PND } & & \multicolumn{2}{c}{ 180 PND } \\
\cline { 2 - 3 } \cline { 6 - 6 } Behavior Latency & Cocaine (6) & Control (5) & & Cocaine (11) & Control (10) \\
\hline Other & $11.55 \pm 4.25$ & $8.92 \pm 4.78$ & & $19.26 \pm 6.54$ & $12.59 \pm 2.81$ \\
Alert posture & $4.15 \pm 2.80$ & $0.44 \pm 0.44$ & & $4.54 \pm 1.87$ & $2.10 \pm 0.62$ \\
Piloerection & $0.00 \pm 0.00$ & $0.00 \pm 0.00$ & & $51.43 \pm 29.02$ & $153.81 \pm 51.59$ \\
Aggressive posture & $101.42 \pm 64.32$ & $0.00 \pm 0.00$ & $103.41 \pm 54.09$ & $122.62 \pm 77.75$ \\
Fight attack & $96.30 \pm 61.34$ & $0.00 \pm 0.00$ & $81.32 \pm 53.41$ & $14.76 \pm 14.76$ \\
Chase & $43.33 \pm 43.33$ & $29.20 \pm 29.20$ & & $136.64 \pm 77.37$ & $632.87 \pm 136.02$ \\
Self-groom & $207.45 \pm 73.08$ & $276.78 \pm 113.02$ & & $49.04 \pm 10.45$ & $203.04 \pm 54.15$ \\
Circle threat & $120.92 \pm 76.56$ & $0.00 \pm 0.00$ & $99.65 \pm 59.53$ & $33.09 \pm 33.09$ \\
Defensive posture & $0.00 \pm 0.00$ & $0.00 \pm 0.00$ & & $21.00 \pm 21.00$ & $32.05 \pm 32.05$ \\
Escape & $117.38 \pm 117.38$ & $136.48 \pm 136.48$ & & $43.58 \pm 32.69$ & $101.59 \pm 47.15$ \\
Rough groom & $12.38 \pm 3.52$ & $10.94 \pm 3.96$ & $15.48 \pm 2.57$ & $25.19 \pm 6.19$ \\
\hline
\end{tabular}

Values are means \pm SEM and the number of subjects per group is shown in parentheses.

* $p<0.01$ 